\title{
Diastereoselective conjugate additions of a D-glucose-derived $\delta$-lactone
}

\author{
Marisa Ciampini ${ }^{1}$, Patrick Perlmutter ${ }^{1} *$ and Keith Watson ${ }^{2}$ \\ ${ }^{1}$ School of Chemistry, Monash University, PO Box 23, Victoria 3800 Australia; ${ }^{2}$ Biota \\ Chemistry Laboratory, Monash University, PO Box 23, Victoria 3800 Australia. \\ E-mail: Patrick.perlmutter@sci.monash.edu.au
}

Dedicated to Professor Sukh Dev on the occasion of his $80^{\text {th }}$ birthday

(received 30 Dec 02; accepted 07 Apr 03; published on the web 02 May 03)

\begin{abstract}
Diastereoselective conjugate additions of a range of nucleophiles, some achieving excellent selectivity, to a D-glucose-derived $\delta$-lactone are reported.
\end{abstract}

Keywords: Conjugate addition, Michael reaction, gluconolactone, diastereoselective

\section{Introduction}

As part of a program directed towards the preparation of a variety of carbon- and heteroatomlinked disaccharides we had cause to investigate the conjugate addition of several nucleophiles to monosaccharide-derived $\delta$-lactones. In this paper we report on additions of three different nucleophiles to a D-glucose-derived $\delta$-lactone.

\section{Results and Discussion}

\section{Preparation of new $\delta$-lactones 7 and 8}

The $\delta$-lactone we chose to study was the differentially protected D-gluconolactone 8 (Scheme 1). This lactone has not been reported previously and so we first needed to develop an efficient synthesis of $\mathbf{8}$. The approach we developed is outlined in Scheme 1. Most of the steps were straightforward providing lactone 8 in five steps and 18\% overall yield from D-glucal triacetate. Differential protection of the three alcohols (at C3, C4 and C6) was required. Monosilylation ${ }^{1,2}$ of the primary alcohol gave ether 3 in excellent yield. Differentiating the two secondary alcohols is less well precedented. Complete selectivity for and acceptable yields $(\sim 60 \%)$ of the monobenzoyl derivative 4 could be achieved by treatment of diol 3 with benzoyl chloride ${ }^{1}$ and DMAP at room temperature in pyridine. Benzylation of $\mathbf{4}$, using potassium hydride ${ }^{2}$ and benzyl 
bromide led to poor yields $(\sim 20 \%)$ of tri-protected 6. A significant improvement in the yield $(70 \%)$ was obtained when the potassium hydride was replaced by silver oxide ${ }^{3}$. Lastly we were pleased to find that oxidative rearrangement ${ }^{4}$ of benzoates $\mathbf{5}$ and $\mathbf{6}$ led smoothly to the new $\delta$ lactones $\mathbf{7}$ and $\mathbf{8}$, respectively.<smiles>CC(=O)OCC1OC=CC(OC(C)=O)C1OC(C)=O</smiles>

1 (i)<smiles>OCC1OC=C[C@@H](O)[C@H]1O</smiles>

2

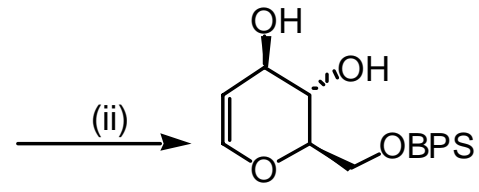

3<smiles>[R]O[Sb]</smiles>

$7 \mathrm{R}=\mathrm{Bz}$

$8 \mathrm{R}=\mathrm{Bn}$ (v)<smiles>[R5]O[C@@H]1C=CO[C@H](CO[SbH2])C1[R]</smiles>

$5 \mathrm{R}=\mathrm{Bz}$

$6 \mathrm{R}=\mathrm{Bn}$

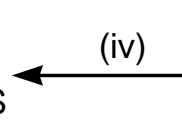<smiles>CCCCO[C@@H]1C=CO[C@@H](CO[SbH2])[C@@H]1O</smiles>

4

Scheme 1. (i) $\mathrm{NaOMe}, \mathrm{MeOH}$; (ii) imidazole, $\mathrm{BPSCl}$, DMF; (iii) $\mathrm{BzCl}$, DMAP, Py; (iv) $\mathrm{BnBr}$, $\mathrm{Ag}_{2} \mathrm{O}$, DMF; (v) MCPBA, BF3.Et2 $\mathrm{O}$.

\section{Conjugate additions to 8}

Conjugate additions ${ }^{5}$ to 8 were then examined (Scheme 2). Three nucleophiles, nitromethane enolate, thiophenolate and benzylamine were chosen for this study as representative C-, S- and $\mathrm{N}$-based nucleophiles.<smiles>O=C1C=C[C@@H](OCc2ccccc2)C(CO[SbH2])O1</smiles>

8

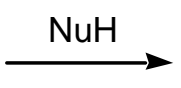

$$
\begin{aligned}
& \text { 9a } \mathrm{Nu}=\mathrm{CH}_{2} \mathrm{NO}_{2} \\
& \text { 10a } \mathrm{Nu}=\mathrm{SPh} \\
& \text { 11a } \mathrm{Nu}=\mathrm{NHBn}
\end{aligned}
$$<smiles>N#CC1CC(=O)OC(CO[SbH2])[C@@H]1OCc1ccccc1</smiles>

9b $\mathrm{Nu}=\mathrm{CH}_{2} \mathrm{NO}_{2}$

$10 \mathrm{~b} \mathrm{Nu}=\mathrm{SPh}$

11b $\mathrm{Nu}=\mathrm{NHBn}$

\section{Scheme 2}

In all cases the diastereoselectivity was significantly influenced by reaction temperature (Table 1). Of the three nucleophiles examined nitromethane and benzylamine gave the most useful results. In both cases conditions were found which gave exclusively a single adduct in 
good to excellent yields. In the former case where $\mathrm{TBAF}^{6}$ was employed as base, the reaction was completely diastereoselective but the product was found to be 9c.

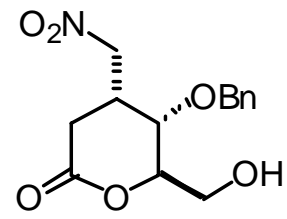

9c

Presumably, although there is no proof at this stage, desilylation occurs after addition of the nitromethane anion. It is of course also possible that desilylated $\mathbf{8}$ may also undergo diastereoselective addition. In any case this is a rather interesting and potentially very useful one-pot conversion as the free alcohol is often required once the addition has been effected. At lower temperatures diastereomeric mixtures were obtained. Only the thiophenolate additions were somewhat disappointing. This is most likely due to the addition being reversible and incomplete at higher temperatures.

Table 1. Results of conjugate additions to $\delta$-lactone 8

\begin{tabular}{|c|c|c|c|c|c|c|}
\hline Entry & Nucleophile & Solvent & $\begin{array}{c}\text { Temp } \\
\left({ }^{\circ} \mathrm{C}\right)\end{array}$ & $\begin{array}{c}\text { Time } \\
\text { (h) }\end{array}$ & $\begin{array}{c}\text { Yield } \\
(\%)\end{array}$ & $\begin{array}{c}\mathrm{Dr} \\
(\mathbf{a}: \mathbf{b})\end{array}$ \\
\hline 1 & $\mathrm{CH}_{3} \mathrm{NO}_{2}{ }^{\mathrm{a}}$ & $\mathrm{CH}_{3} \mathrm{CN}$ & Reflux & 1 & 73 & $6.2^{\mathrm{b}}: 1$ \\
\hline 2 & $\mathrm{CH}_{3} \mathrm{NO}_{2}$ & $\mathrm{CH}_{3} \mathrm{CN}$ & Rt & $4 d^{c}$ & $<20$ & $5: 1$ \\
\hline 3 & $\mathrm{CH}_{3} \mathrm{NO}_{2}{ }^{\mathrm{d}}$ & $\mathrm{CH}_{3} \mathrm{CN}$ & Reflux & 0.33 & $66^{\mathrm{e}}$ & $100: 0$ \\
\hline 4 & $\mathrm{PhSH}^{\mathrm{d}}$ & THF & $\mathrm{Rt}$ & 1 & $60^{f}$ & $3: 1$ \\
\hline 5 & $\mathrm{PhSH}^{\mathrm{d}}$ & THF & Reflux & 1 & 60 & $3: 1$ \\
\hline 6 & $\mathrm{PhSH}^{\mathrm{d}}$ & $\mathrm{THF}$ & Reflux & 1 & $80^{\mathrm{g}}$ & $3: 1$ \\
\hline 7 & $\mathrm{BnNH}_{2}$ & $\mathrm{MeOH}$ & $-78^{\circ} \mathrm{C}$ & $18 \mathrm{~d}^{\mathrm{c}}$ & $41^{\mathrm{h}}$ & $1: 5$ \\
\hline 8 & $\mathrm{BnNH}_{2}$ & $\mathrm{MeOH}$ & $\mathrm{Rt}$ & 24 & 99 & $3: 2$ \\
\hline 9 & $\mathrm{BnNH}_{2}$ & $\mathrm{MeOH}$ & Reflux & 8 & 92 & $100: 0$ \\
\hline
\end{tabular}

a. Base used was $\mathrm{KF}^{7}$ in the presence of 18 -crown- $6^{8}$; b. $18 \%$ of 9a obtained as desilylated product 9c; c. Note the time is days not hours for this entry; d. TBAF was used as base in these experiments; e. Product obtained was exclusively 9c; f. At 85\% conversion; g. crude yield; h. At $70 \%$ conversion.

From these results each of the nucleophiles appear to prefer addition to the same face of $\mathbf{8}$ as shown in the Scheme below. This selectivity can be explained by assuming that the chair-like transition state is lower in energy than the boat-like transition state which would follow from addition to the opposite face of $\mathbf{8}$. This outcome is similar to that reported by Herradon et $\mathrm{al}^{9}$ in the conjugate addition of carbon nucleophiles to lactone $\mathbf{1 0 .}$ 


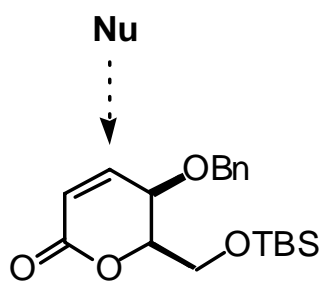

10

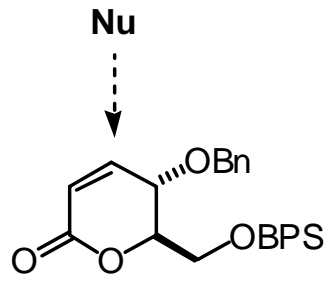

8

\section{Conclusions}

A concise and straightforward synthesis of the new $\delta$-lactone 8 is reported. Conjugate additions of C-, N- and S-based nucleophiles were examined and conditions were found for the first two nucleophiles which led exclusively to the adducts 9a and 11a. In contrast addition of thiophenolate showed poorer selectivity.

\section{Acknowledgements}

M.C. thanks the Australian Research Council for an Australian Post-Graduate Award (Industry) and Biota Holdings Inc. for financial support

\section{Experimental Section}

General Procedures. Melting points were determined on a Kofler hotstage and are uncorrected. Elemental microanalyses were performed by the Australian Microanalytical Service, National Analytical Laboratories, Melbourne or the University of Otago, Dunedin, New Zealand. Optical rotations were recorded on a Perkin Elmer Model 141 Polarimeter. Infrared (IR) spectra were recorded on a Perkin-Elmer 1600 Series Fourier Transform spectrophotometer $\left(\mathrm{cm}^{-1} \mathrm{scale}\right)$ and refer to thin films of liquids (neat) or paraffin (Nujol) mulls of solids between $\mathrm{NaCl}$ plates. High resolution hydrogen-1 nuclear magnetic resonance ( $\left.{ }^{1} \mathrm{H} \mathrm{NMR}\right)$ spectra were recorded at $300 \mathrm{MHz}$ on a Bruker DPX-300 spectrometer or $400 \mathrm{MHz}$ on a Bruker Avance DRX 400 spectrometer. The ${ }^{1} \mathrm{H}$ NMR spectral data refer to deuteriochloroform solutions $\left(\mathrm{CDCl}_{3}\right)$ using tetramethylsilane (TMS) as internal reference $(\delta 0.00 \mathrm{ppm})$. Carbon-13 nuclear magnetic resonance $\left({ }^{13} \mathrm{C} \mathrm{NMR}\right)$ spectra were recorded at $75 \mathrm{MHz}$ on a Bruker APX-300 spectrometer or $100 \mathrm{MHz}$ on a Bruker Avance DRX 400 spectrometer. Mass spectrometry (ESI) was performed using samples in methanol on a Micromass Platform QMS Electrospray mass spectrometer. High resolution mass spectra (HRMS) for accurate mass determinations were recorded on a Bruker BioApex 47e FTMS fitted with an Analytica electrospray source using NaI for accurate mass calibration 
(accuracy $\pm 3 \mathrm{ppm}$ ). Low resolution mass spectra were recorded on a VG micromass $70 / 70 \mathrm{~F}$ or a VG TRIO-1 mass spectrometer with an ion source temperature of $200^{\circ}$ and electron impact energy of $70 \mathrm{eV}$.

1,5-Anhydro-6-O-t-butyldiphenylsilyl-2-deoxy-D-arabino-hex-1-enitol (3). A solution of the triol $2^{1,2}(2.04 \mathrm{~g}, 14.0 \mathrm{mmol})$ dissolved in DMF $(20.0 \mathrm{ml})$ was treated with imidazole $(2.08 \mathrm{~g}$, $30.59 \mathrm{mmol})$ followed by BPSCl $(4.28 \mathrm{~g}, 4.05 \mathrm{ml}, 15.6 \mathrm{mmol})$. The mixture was stirred at room temperature for $16 \mathrm{hr}$, diluted with $\mathrm{Et}_{2} \mathrm{O}(100 \mathrm{ml})$ and the resulting solution washed with water $(50.0 \mathrm{ml})$, saturated $\mathrm{CuSO}_{4}$ solution $(50 \mathrm{ml})$ and water $(2 \mathrm{X} 50 \mathrm{ml})$. The organic extracts were combined, dried $\left(\mathrm{MgSO}_{4}\right)$ and concentrated. The crude product was subjected to purification by flash column chromatography $\left(\mathrm{SiO}_{2} \quad 3: 2\right.$ light petroleum/ethyl acetate) to yield the title compound 3 as a clear oil $\left(4.34 \mathrm{~g}, \mathrm{R}_{\mathrm{f}} 0.34,81 \%\right)$. IR $\left(\mathrm{CH}_{2} \mathrm{Cl}_{2}\right): 3421 \mathrm{~s}, 3054 \mathrm{~s}, 2931 \mathrm{~s}, 2858 \mathrm{~s}$, $1718 \mathrm{w}, 1648 \mathrm{~s}, 1472 \mathrm{~m}, 1428 \mathrm{~s}, 1391 \mathrm{w}, 1265 \mathrm{~s}, 1112 \mathrm{~s}, 926 \mathrm{w}, 896 \mathrm{w}, 877 \mathrm{w}, 823 \mathrm{w}, 739 \mathrm{~s}, 703 \mathrm{~s} \mathrm{~cm}^{-1}$. ${ }^{1} \mathrm{H}$ n.m.r. $\delta 1.07$, s, 9H, 3( $\left.\mathrm{CH}_{3}\right)$-BPS; 3.93-3.78, m, 2H, 2(CH), H4, H5; 4.01, dq, J 10.1, 4.00, $2.80 \mathrm{~Hz}, 2 \mathrm{H}, \mathrm{CH}_{2}, \mathrm{H} 6 ; 4.29$, td, J 7.00, $4.00 \mathrm{~Hz}, 1 \mathrm{H}, \mathrm{CH}, \mathrm{H} 3 ; 4.73$, dd, J 6.1, $2.2 \mathrm{~Hz}, 1 \mathrm{H}, \mathrm{CH}$, H2; 6.32, dd, J 6.0, $1.7 \mathrm{~Hz}, 1 \mathrm{H}, \mathrm{CH}, \mathrm{H} 1 ; 7.48-7.37, \mathrm{~m}, 6 \mathrm{ArH}$-BPS; 7.67-7-71, m, 4ArH -BPS. ${ }^{13} \mathrm{C}$ n.m.r. $\delta .19 .35, \mathrm{CH}_{2}, \mathrm{C} 6 ; 26.87,3\left(\mathrm{CH}_{3}\right)$-BPS; 63.77, $\underline{\mathrm{C}}\left(\mathrm{CH}_{3}\right)$-BPS; 69.60, $\mathrm{CH}, \mathrm{C} 5 ; 71.57$, $\mathrm{CH}, \mathrm{C} 3 ;$ 77.15, CH, C4; 102.40, CH, C2; 127.66, 127.71, ArCH, 2(C2', C6') -BPS; 129.76, 129.79, $\mathrm{ArCH}, 2(\mathrm{C} 4 ')$-BPS; 132.63, 132.83, $\mathrm{ArC}, 2\left(\mathrm{C} 1^{\prime}\right)$-BPS; 135.42, 135.52, $\mathrm{ArCH}, 2(\mathrm{C} 3$ ', C6') -BPS; 144.11, CH, C1. MS: $407.2\left([\mathrm{M}+\mathrm{Na}]^{+}, 100 \%\right), 423.1,\left([\mathrm{M}+\mathrm{K}]^{+}, 58\right), 791.3$, ([2M + $\left.\mathrm{Na}]^{+}, 90\right), 807.1,\left([2 \mathrm{M}+\mathrm{K}]^{+}, 32\right)$. HRMS calc'd for $\mathrm{C}_{22} \mathrm{H}_{28} \mathrm{O}_{4} \mathrm{Si}\left(\mathrm{Na}^{+}\right)$requires 407.165 found 407.165. Anal. Calc'd for $\mathrm{C}_{22} \mathrm{H}_{28} \mathrm{O}_{4} \mathrm{Si}$ (384.2): C, 68.72; H, 7.34. Found C, 68.96; H, 6.92. $[\alpha]_{D}^{24}$ $+4.93\left(\mathrm{c}=1.5, \mathrm{CHCl}_{3}\right)$.

1,5-Anhydro-3-O-benzoyl-6-O-t-butyldiphenylsilyl-2-deoxy-D-arabino-hex-1-enitol (4). To a cool $\left(-35^{\circ}\right)$ solution of the monosilylated diol $3(2.00 \mathrm{~g}, 5.20 \mathrm{mmol})$ in pyridine $(9.0 \mathrm{ml}) \mathrm{a}$ solution of 4-(dimethylamino)pyridine $(0.06 \mathrm{~g}, 0.52 \mathrm{mmol})$ in pyridine $(10 \mathrm{ml})$ was added. Neat benzoyl chloride $\left(800 \mathrm{mg}, 0.67 \mathrm{ml}, 5.70 \mathrm{mmol}\right.$ ) was added and the reaction was stirred at $-35^{\circ}$ for $15 \mathrm{~min}$. The reaction was warmed to $0^{\circ}$ over a period of $3 \mathrm{hr}$ and allowed to warm to room temperature overnight $\left(16^{\circ}\right)$. The reaction mixture was poured into $\mathrm{Et}_{2} \mathrm{O}(50 \mathrm{ml})$, washed with water $(50 \mathrm{ml})$, saturated $\mathrm{CuSO}_{4}$ solution $(50 \mathrm{ml})$, followed by water $(50 \mathrm{ml})$ and the combined aqueous phases were reextracted with $\mathrm{Et}_{2} \mathrm{O}(2 \mathrm{X} 40 \mathrm{ml})$. The organic extracts were combined, dried $\left(\mathrm{MgSO}_{4}\right)$ and evaporated under reduced pressure. The residue was purified by flash column chromatography $\left(\mathrm{SiO}_{2}, 5: 1\right.$, light petroleum/ethyl acetate) to give the product 4 as a colourless oil. Recrystallisation of the crude oil from light petroleum gave the desired di-protected sugar 4 (1.52 g, 60\%, $\mathrm{R}_{\mathrm{f}} 0.34$ ), as colourless crystals (m.p. $\left.78.5-80^{\circ}\right)$. IR $\left(\mathrm{CH}_{2} \mathrm{Cl}_{2}\right): 3448 \mathrm{~m}, 3054 \mathrm{~m}$, $2933 \mathrm{~m}, 2859 \mathrm{w}, 1718 \mathrm{~m}, 1648 \mathrm{w}, 1602 \mathrm{w}, 1473 \mathrm{w}, 1452 \mathrm{w}, 1428 \mathrm{~m}, 1309 \mathrm{w}, 1316 \mathrm{~m}, 1285 \mathrm{~s}, 1178 \mathrm{w}$, $1113 \mathrm{~m}, 1071 \mathrm{w}, 1060 \mathrm{w}, 1027 \mathrm{w}, 896 \mathrm{w} \mathrm{cm}{ }^{-1} .{ }^{1} \mathrm{H}$ n.m.r. $\delta .1 .06$, s, 9H, 3( $\left.\mathrm{CH}_{3}\right)$-BPS; 3.25-3.40, bs, $1 \mathrm{H}, \mathrm{OH} ; 3.95-4.12, \mathrm{~m}, 3 \mathrm{H}, 1\left(\mathrm{CH}_{2}\right), \mathrm{H} 6$ and $1(\mathrm{CH}), \mathrm{H} 4 ; 4.24$, dd, J 3.00, $4.02 \mathrm{~Hz}, 1 \mathrm{H}, \mathrm{CH}, \mathrm{H} 5$; 4.83 , dd, $J_{2 \mathrm{a}-3} 2.6 \mathrm{~Hz}, J_{2 \mathrm{e}-3} 2.6 \mathrm{~Hz}, 1 \mathrm{H}, \mathrm{CH}, \mathrm{H} 2 ; 5.53$, dq, $J 1.5,2.6 \mathrm{~Hz}, 1 \mathrm{H}, \mathrm{CH}, \mathrm{H} 3 ; 6.48$, dd $J$ 6.0, $1.4 \mathrm{~Hz}, 1 \mathrm{H}, \mathrm{CH}, \mathrm{H1}$; 7.35-7.47, m, 8ArH; 7.53-7.59, m, 1ArH, (H4') -Bz; 7.68-7.73, m, 4ArH; 8.03-8.07, m, 2ArH, (H2', H6') -Bz. ${ }^{13} \mathrm{C}$ n.m.r. $\delta .19 .40, \mathrm{CH}_{2}, \mathrm{C} 6 ; 26.89,3\left(\mathrm{CH}_{3}\right)$-BPS; 
63.01, $\underline{\mathrm{C}}\left(\mathrm{CH}_{3}\right)_{3}$-BPS; 68.07, $\mathrm{CH}, \mathrm{C} 5 ; 73.44, \mathrm{CH}, \mathrm{C} 3 ; 78.05, \mathrm{CH}, \mathrm{C} 4 ; 98.59, \mathrm{CH}, \mathrm{C} 2 ; 127.62$, 127.65, $\mathrm{ArCH}, 2\left(\mathrm{C} 2^{\prime}, \mathrm{C}^{\prime}\right)$ - $\mathrm{BPS} ; 128.28, \mathrm{ArCH}, 2\left(\mathrm{C} 2^{\prime}, \mathrm{C}^{\prime}\right)$-Bz; 129.70, $\mathrm{ArCH}, 2(\mathrm{C} 4 ')$-BPS, Bz; 132.95, 133.01, ArC, 2(C1') -BPS, -Bz; 133.15, ArCH, 2(C3', C5') -Bz; 135.48, 135.52, $\mathrm{ArCH},\left(\mathrm{C} 3 ', \mathrm{C}^{\prime}\right)$-BPS; 146.18, CH, C1; 167.31, CO. MS: 511.19 ([M + Na $\left.]^{+}, 29 \%\right), 999.41$, $\left([2 \mathrm{M}+\mathrm{Na}]^{+}, 72\right)$. HRMS calc'd for $\mathrm{C}_{29} \mathrm{H}_{32} \mathrm{O}_{5} \mathrm{Si}\left(\mathrm{Na}^{+}\right)$requires 511.192 found 511.192. Anal.

Calc'd for $\mathrm{C}_{29} \mathrm{H}_{32} \mathrm{O}_{5} \mathrm{Si}$ (488.2): C,71.28; H, 6.60. Found C, 71.46; H, 6.41. $[\alpha]_{\mathrm{D}}^{25}-57.5^{\circ}(\mathrm{c}=1$, $\mathrm{CHCl}_{3}$ ).

\section{1,5-Anhydro-3-O-benzoyl-4-O-benzyl-6-O-t-butyldiphenylsilyl-2-deoxy-D-arabino-hex-1- enitol (5).}

\section{Method 1}

A solution of the alcohol 4 (500 mg, $1.02 \mathrm{mmol})$ in THF $(5 \mathrm{ml})$ was added to a stirred slurry of $\mathrm{KH}\left(35 \%\right.$ dispersion in oil, oil not removed) $(0.07 \mathrm{~g}, 1.63 \mathrm{mmol})$ at $0^{\circ}$. The resulting solution was stirred for $15 \mathrm{~min}$, neat benzyl bromide $(0.28 \mathrm{~g}, 0.19 \mathrm{ml}, 1.61 \mathrm{mmol})$ added and stirred for a further $2 \mathrm{hr}$ at $0^{\circ}$. The contents were added to water $(50 \mathrm{ml})$, and the aqueous phase extracted with $\mathrm{Et}_{2} \mathrm{O}(3 \mathrm{X} 40 \mathrm{ml})$. The organic extracts were combined, dried $\left(\mathrm{MgSO}_{4}\right)$ and evaporated under reduced pressure. Purification by flash column chromatography $\left(\mathrm{SiO}_{2}, 7 \%\right.$ ethyl acetate in light petroleum) yielded two products $6\left(100 \mathrm{mg}, 17 \%, \mathrm{R}_{\mathrm{f}} 0.3\right), 5(52 \mathrm{mg}, 9 \%$, Rf 0.26$)$ both isolated as colourless oils, as well as recovered 4 (216 mg, $\left.\mathrm{R}_{\mathrm{f}} 0.09\right) .6 \mathrm{IR}\left(\mathrm{CH}_{2} \mathrm{Cl}_{2}\right): 3053 \mathrm{~m}$, 2946s, 2930s, 2857s, 1740m, 1719s, 1649m, 1604w, 1560w, 1584w, 1494w, 1464w, 1452m, $1428 \mathrm{~m}, 1390 \mathrm{~m}, 1286 \mathrm{~s}, 1243 \mathrm{~m}, 1172 \mathrm{w}, 1107 \mathrm{~s}, 1067 \mathrm{~m}, 1026 \mathrm{~m}, 941 \mathrm{w}, 821 \mathrm{~m}, 792 \mathrm{~m}, 738 \mathrm{~s}, 702 \mathrm{~s}$ $\mathrm{cm}^{-1} .{ }^{1} \mathrm{H}$ n.m.r. $\delta 1.02$, s, $9 \mathrm{H}, 3\left(\mathrm{CH}_{3}\right) ; 3.94-4.02, \mathrm{~m}, 2 \mathrm{H}, \mathrm{CH}_{2}, \mathrm{H} 6$; 4.04-4.13, dd, J 7.0, $5.4 \mathrm{~Hz}$, 1H, CH, H4; 4.22, m, 1H, CH, H5; 4.75, 4.78, ABq, J $1.47 \mathrm{~Hz}, 2 \mathrm{H}, \mathrm{CH}_{2}-\mathrm{Ph} ; 4.86$, dd, J 3.09, $6.11 \mathrm{~Hz}, 1 \mathrm{H}, \mathrm{CH}, \mathrm{H} 2$; 5.62-5.65, m, 1H, CH, H3; 6.45, dd, J 1.31, $6.0 \mathrm{~Hz}, 1 \mathrm{H}, \mathrm{CH}, \mathrm{H1} ; 7.21-$ 7.23, m, 4ArH; 7.29-7.71, m, 8ArH; 7.51-7.57, m, 2ArH; 7.61-7.66, m, 4ArH; 7.96-7.99, m, 2ArH. ${ }^{13} \mathrm{C}$ n.m.r. $\delta 19.34, \mathrm{CH}_{2}, \mathrm{C} 6 ; 26.90,3\left(\mathrm{CH}_{3}\right)$-BPS; 61.80, $\underline{\mathrm{C}}\left(\mathrm{CH}_{3}\right)_{3}-\mathrm{BPS} ; 70.24, \mathrm{CH}, \mathrm{C} 5$; 72.90, CH, C3; 73.37, $\underline{\mathrm{CH}}_{2}$-Bn; 77.57, CH, C4; 98.37, CH, C2; 127.52, 127.60, ArCH, 2(C2', C6') -BPS; 127.70, ArC, (C2', C6') -Bn; 128.26, ArC, 2(C2', C6') -Bz; 129.50, ArCH, (C4') -Bn, $\mathrm{Bz}$; 129.58, $\mathrm{ArCH}, 2(\mathrm{C} 4 ')$-BPS; 130.00, $\mathrm{ArC},\left(\mathrm{Cl}^{\prime}\right)$-Bz; 132.90, $\mathrm{ArCH}, 2(\mathrm{C} 3, \mathrm{C} 5 ')-\mathrm{Bn},-\mathrm{Bz}$; 133.25, ArC, (C1') -BPS; 135.46, 135.59, $\mathrm{ArCH}, 2\left(\mathrm{C} 3 ', \mathrm{C} 5{ }^{\prime}\right)$-BPS; 137.70, $\mathrm{ArC},\left(\mathrm{C} 1{ }^{\prime}\right)$-Bn; 145.90, CH, C1; 165.89, CO. MS: $\left.601.24[\mathrm{M}+\mathrm{Na}]^{+}, 72 \%\right), 617.21$, $\left([\mathrm{M}+\mathrm{K}]^{+}, 10\right), 1179.51$, $\left([2 \mathrm{M}+\mathrm{Na}]^{+}\right.$, 52). HRMS calc'd for $\mathrm{C}_{36} \mathrm{H}_{38} \mathrm{O}_{5} \mathrm{Si}\left(\mathrm{Na}^{+}\right)$requires 601.239 found 601.238. $\mathrm{C}_{36} \mathrm{H}_{38} \mathrm{O}_{5} \mathrm{Si}\left(\mathrm{K}^{+}\right)$requires 617.213 found 617.211. Anal. Calc'd for $\mathrm{C}_{36} \mathrm{H}_{38} \mathrm{O}_{5} \mathrm{Si}$ (578.2): C, 74.71; $\mathrm{H}, 6.61$. Found $\mathrm{C}, 75.00 ; \mathrm{H}, 6.81 .[\alpha]_{\mathrm{D}}^{23}-34.2^{\circ}\left(\mathrm{c}=1.5, \mathrm{CHCl}_{3}\right.$.

1,5-Anhydro-3,4-bis-O-benzoyl-6-O-t-butyldiphenylsilyl-2-deoxy-D-arabino-hex-1 enitol (5). (52 mg, 9\%, $\mathrm{R}_{\mathrm{f}}$ 0.26). IR $\left(\mathrm{CH}_{2} \mathrm{Cl}_{2}\right): 3054 \mathrm{~m}, 2960 \mathrm{~m}, 2932 \mathrm{~m}, 2891 \mathrm{~m}, 2859 \mathrm{~m}, 1968 \mathrm{w}, 1911 \mathrm{w}$, $1720 \mathrm{~s}, 1648 \mathrm{~s}, 1602 \mathrm{~m}, 1587 \mathrm{~m}, 1490 \mathrm{w}, 1473 \mathrm{~m}, 1452 \mathrm{~s}, 1428 \mathrm{~s}, 1391 \mathrm{~m}, 1362 \mathrm{~m}, 1316 \mathrm{~s}, 1265 \mathrm{~s}$, $1178 \mathrm{~m}, 1158 \mathrm{~m}, 1108 \mathrm{~s}, 1070 \mathrm{~m}, 1027 \mathrm{~m}, 998 \mathrm{~m}, 941 \mathrm{~m}, 896 \mathrm{~m}, 879 \mathrm{w}, 850 \mathrm{w}, 824 \mathrm{~m} \mathrm{~cm}{ }^{-1} .{ }^{1} \mathrm{H}$ n.m.r. $\oint 1.02$, s, 9H, 3( $\left.\mathrm{CH}_{3}\right)$-BPS; 3.96, d, J $4.6 \mathrm{~Hz}, \mathrm{CH}_{2}, 2 \mathrm{H}, \mathrm{H6} ; 4.36$, dq, J 4.70, $11.2 \mathrm{~Hz}, 1 \mathrm{H}, \mathrm{CH}$, H5; 4.99, dd, J 6.20, $3.40 \mathrm{~Hz}, 1 \mathrm{H}, \mathrm{CH}, \mathrm{H} 2 ; 5.60$, q, J 5.01, $3.90 \mathrm{~Hz}, 1 \mathrm{H}, \mathrm{H} 3 ; 5.82$, t, J 6.6, 5.3 
$\mathrm{Hz}, 1 \mathrm{H}, \mathrm{CH}, \mathrm{H} 4 ;$ 6.51, dd, J 6.1, 1.0 Hz, 1H, CH, H1; 7.17-7.68, m, 16 ArH; 7.92-8.01, 4ArH, 2(H2', H6') -Bz. ${ }^{13} \mathrm{C}$ n.m.r. $\delta 19.26, \mathrm{CH}_{2}, \mathrm{C} 6 ; 26.76,3\left(\mathrm{CH}_{3}\right)$-BPS; 61.72, $\underline{\mathrm{C}}\left(\mathrm{CH}_{3}\right)$-BPS; 67.64, $\mathrm{CH}, \mathrm{C} 5 ; 67.91, \mathrm{CH}, \mathrm{C} 3 ;$ 76.70, CH, C4; 98.16, CH, C2; 127.51, 127.59, ArCH, 2(C2', C6') -BPS; 128.25, 128.31, ArCH, 2(C2', C6') -Bz; 129.49, ArC, (C1') -Bz; 129.57, 129.63, ArCH, 2(C4') $\mathrm{Bz} ; 129.73, \mathrm{ArCH}, 2\left(\mathrm{C} 4^{\prime}\right)$-BPS, -Bz; 132.89, ArC, (C1') -BPS; 132.93, 132.13, ArCH, 2(C3', C5') -Bz; 135.45, 135.47, ArCH, 2(C3', C5') -BPS; 146.00, CH, C1; 164.74, 165.74, 2(CO). MS: $615.21\left([\mathrm{M}+\mathrm{Na}]^{+}, 45 \%\right), 1207.45\left([2 \mathrm{M}+\mathrm{Na}]^{+}, 72\right)$. HRMS calc'd for $\mathrm{C}_{36} \mathrm{H}_{36} \mathrm{O}_{6} \mathrm{Si}\left(\mathrm{Na}^{+}\right)$requires 615.218 found 615.216. Anal. Calc'd for $\mathrm{C}_{36} \mathrm{H}_{36} \mathrm{O}_{6} \mathrm{Si}$ (592.2): C, 72.40; H, 6.44. Found C, 72.58; $\mathrm{H}, 6.21 .[\alpha]_{\mathrm{D}}^{22}+15.5^{\circ}\left(\mathrm{c}=2.7, \mathrm{CHCl}_{3}\right.$.

\section{Method 2}

A solution of the alcohol $4(200 \mathrm{mg}, 0.41 \mathrm{mmol})$ in THF $(30.0 \mathrm{ml})$ at $0^{\circ}$ was added to a stirred slurry of $\mathrm{KH}$ (35\% dispersion in oil, oil not removed) $(0.03 \mathrm{~g}, 0.65 \mathrm{mmol})$ in THF (8.0 ml) also at $0^{\circ}$. The resulting solution was stirred for $15 \mathrm{~min}$, neat benzyl bromide $(0.11 \mathrm{~g}, 0.08 \mathrm{ml}, 0.65$ mmol) added and left overnight $(15 \mathrm{hr})$ in the fridge $\left(3^{\circ}\right)$. The contents was added to water $(50$ $\mathrm{ml})$, and the aqueous phase extracted with $\mathrm{Et}_{2} \mathrm{O}(3 \mathrm{X} 40 \mathrm{ml})$. The organic phases were combined, dried $\left(\mathrm{MgSO}_{4}\right)$ and evaporated under reduced pressure. Purification by flash column chromatography $\left(\mathrm{SiO}_{2}, 7 \%\right.$ ethyl acetate in light petroleum) gave firstly the desired compound 6 as a colourless oil $(63.0 \mathrm{mg}, 27 \%)$. The column was flushed with ethyl acetate to obtain $140 \mathrm{mg}$ of an unknown mixture. Purification of this material by flash column chromatography $\left(\mathrm{SiO}_{2}, 3: 2\right.$, light petroleum/ethyl acetate) gave $(2 R, 3 R, 4 R)-4-((t e r t-b u t y l d i p h e n y l s i l y l) o x y)-2-$ (hydroxymethyl)-3-(hydroxy)-3,4-dihydro-2H-pyran 12 (24.8 mg, 18\%) and diol 3 (70.1 mg, $41 \%)$.

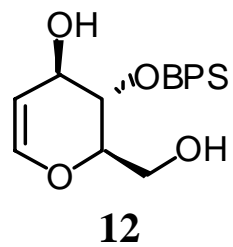

12 IR $\left(\mathrm{CH}_{2} \mathrm{Cl}_{2}\right): 3385 \mathrm{~s}, 3071 \mathrm{~m}, 2931 \mathrm{~s}, 2857 \mathrm{~s}, 1708 \mathrm{w}, 1647 \mathrm{~s}, 1590 \mathrm{w}, 1472 \mathrm{~m}, 1428 \mathrm{~s}, 1390 \mathrm{w}$, $1362 \mathrm{w}, 1326 \mathrm{w}, 1235 \mathrm{~s}, 1170 \mathrm{w}, 1113 \mathrm{~s}, 1053 \mathrm{~s}, 949 \mathrm{w}, 875 \mathrm{w}, 823 \mathrm{~m}, 798 \mathrm{~m}, 740 \mathrm{~s}, 702 \mathrm{~s} \mathrm{~cm}{ }^{-1} .{ }^{1} \mathrm{H}$ n.m.r. $\delta 1.05, \mathrm{~s}, 9 \mathrm{H}, 3\left(\mathrm{CH}_{3}\right)$-BPS; 1.64, bs, OH; 2.91, bs, OH; 3.74-3.97, m, 4H, 2(CH), H4, H5, $1\left(\mathrm{CH}_{2}\right), \mathrm{H} 6 ; 4.16-4.18, \mathrm{~m}, 1 \mathrm{H}, \mathrm{CH}, \mathrm{H} 3 ; 4.71$, dd, J 2.11, $6.03 \mathrm{~Hz}, 1 \mathrm{H}, \mathrm{CH}, \mathrm{H} 2 ; 6.30$, dd, J 1.56, $6.03 \mathrm{~Hz}, 1 \mathrm{H}, \mathrm{CH}, \mathrm{H1} ; 7.38-7.47, \mathrm{~m}, 6 \mathrm{ArH}$-BPS; 7.65-7-69, m, 4ArH -BPS. ${ }^{13} \mathrm{C}$ n.m.r. $\delta .26 .90$, $3\left(\mathrm{CH}_{3}\right)$-BPS; 29.78, $\mathrm{CH}_{2}, \mathrm{C} 6 ; 63.91, \underline{\mathrm{C}}\left(\mathrm{CH}_{3}\right)_{3}$-BPS; 69.58, CH, C5; 71.90, CH, C3; 76.88, CH, $\mathrm{C} 4 ; 102.27, \mathrm{CH}, \mathrm{C} 2 ; 127.71,127.78, \mathrm{ArCH}, 2\left(\mathrm{C}^{\prime}, \mathrm{C} 6{ }^{\prime}\right)$-BPS; 129.84, 129.88, ArCH, 2(C4') BPS; 132.62, 132.83, ArC, 2(C1') -BPS; 135.42, 135.52, ArCH, 2(C3', C6') -BPS; 144.11, CH, C1.

\section{Method 3}

Freshly prepared silver oxide ${ }^{10}(1.02 \mathrm{~g}, 4.4 \mathrm{mmol})$ and benzyl bromide $(0.36 \mathrm{~g}, 0.26 \mathrm{ml}, 2.16$ $\mathrm{mmol})$ were added to a cool $\left(0^{\circ}\right)$ solution of $4(0.43 \mathrm{~g}, 0.879 \mathrm{mmol})$ in DMF $(5 \mathrm{ml})$ under 
nitrogen. The resulting mixture was stirred at $0^{\circ}$ for $15 \mathrm{~min}$, allowed to warm to room temperature and stirred for 2 days. It was diluted with $\mathrm{CH}_{2} \mathrm{Cl}_{2}(10.0 \mathrm{ml})$, filtered and the filtrate concentrated under reduced pressure. The resulting oil was dissolved in $10 \%$ aq. citric acid (10.0 $\mathrm{ml})$ and extracted with $\mathrm{Et}_{2} \mathrm{O}(2 \mathrm{X} 15 \mathrm{ml})$. The organic phases was washed with $10 \%$ aq. $\mathrm{NaHCO}_{3}(10 \mathrm{ml})$ followed by water $(10 \mathrm{ml})$. The organic phase were combined, dried $\left(\mathrm{MgSO}_{4}\right)$ and concentrated under reduced pressure to give an orange oil (1.91 g crude). Purification by flash column chromatography $\left(\mathrm{SiO}_{2}, 20: 1\right.$ light petroleum/ethyl acetate) gave $\mathbf{6}$ as a light orange oil ( $\left.\mathrm{R}_{\mathrm{f}} 0.81,285 \mathrm{mg}, 56 \%\right)$.

\section{1,5-Anhydro-4-O-benzyl-6-O-t-butyldipnenylsilyl-2,3-deoxy-1-oxo-D-arabino-hex-2-enitol}

(8). A solution of MCPBA (85\% anhydrous, $35.2 \mathrm{mg}, 0.21 \mathrm{mmol})$ in $\mathrm{CH}_{2} \mathrm{Cl}_{2}(3 \mathrm{ml})$ was cooled to $-20^{\circ}$ and added to an equally cooled solution of $6(100 \mathrm{mg}, 0.17 \mathrm{mmol})$ in $\mathrm{CH}_{2} \mathrm{Cl}_{2}(3 \mathrm{ml})$. $\mathrm{BF}_{3} . \mathrm{Et}_{2} \mathrm{O}(11.6 \mu \mathrm{L}, 12.98 \mathrm{mg}, 0.092 \mathrm{mmol})$ was then added dropwise. The reaction was stirred at $-20^{\circ}$ for $3.5 \mathrm{hr}$ and quenched with saturated $\mathrm{NaHCO}_{3}$ solution $(10 \mathrm{ml})$. The aqueous phase was reextracted with $\mathrm{CH}_{2} \mathrm{Cl}_{2}(3 \mathrm{X} 10 \mathrm{ml})$ and the organic phases were combined, dried $\left(\mathrm{MgSO}_{4}\right)$ and evaporated under reduced pressure to afford an orange solid (107 $\mathrm{mg}$ crude). Purification by column chromatography $\left(\mathrm{SiO}_{2}, \mathrm{CH}_{2} \mathrm{Cl}_{2}\right)$ gave the desired product 8 as an orange oil $(52.0 \mathrm{mg}$, $64 \%)$. IR $\left(\mathrm{CH}_{2} \mathrm{Cl}_{2}\right): 3071 \mathrm{~s}, 2931 \mathrm{~s}, 2858 \mathrm{~s}, 1962 \mathrm{w}, 1895 \mathrm{w}, 1820 \mathrm{w}, 1738 \mathrm{~s}, 1629 \mathrm{w}, 1589 \mathrm{w}, 1497 \mathrm{w}$, $1472 \mathrm{~m}, 1455 \mathrm{~m}, 1428 \mathrm{~s}, 1390 \mathrm{~s}, 1307 \mathrm{~m}, 1260 \mathrm{~m}, 1228 \mathrm{~m}, 1113 \mathrm{~s}, 1029 \mathrm{~m}, 961 \mathrm{~m}, 857 \mathrm{~m}, 823 \mathrm{~s}, 740 \mathrm{~s}$ $\mathrm{cm}^{-1} .{ }^{1} \mathrm{H}$ n.m.r. $\delta$ (400 MHz) 1.06, s, 9H, 3( $\left.\mathrm{CH}_{3}\right)$-BPS; 3.92, dq, J 11.44, $3.45 \mathrm{~Hz}, 2 \mathrm{H}, \mathrm{CH}_{2}$, H6; 4.43, dt, J 3.64, 6.58 Hz, 1H, CH, H5; 4.54, dq, J 7.60, $2.80 \mathrm{~Hz}, J_{4-2} 1.64 \mathrm{~Hz}, 1 \mathrm{H}, \mathrm{CH}, \mathrm{H} 4 ; 4.64$, q, $J 11.53 \mathrm{~Hz}, 2 \mathrm{H}, \mathrm{CH}_{2}-\mathrm{Ph} ; 5.99$, dd, $J 10.0,1.60 \mathrm{~Hz}, 1 \mathrm{H}, \mathrm{CH}, \mathrm{H} 2 ; 6.82$, dd, $J 10.0,2.7 \mathrm{~Hz}, 1 \mathrm{H}$, $\mathrm{CH}, \mathrm{H} 3$; 7.25-7.27, m, 9ArH; 7.61-7.68, m, 6ArH. ${ }^{13} \mathrm{C}$ n.m.r. $\delta 19.78, \mathrm{CH}_{2}, \mathrm{C} 6 ; 27.31,3\left(\mathrm{CH}_{3}\right)-$ BPS; 62.67, $\underline{\mathrm{C}}\left(\mathrm{CH}_{3}\right)_{3}$-BPS; 68.87, $\mathrm{CH}, \mathrm{C} 5 ; 72.50, \underline{\mathrm{CH}}_{2}-\mathrm{Bn} ; 81.42, \mathrm{CH}, \mathrm{C} 4 ; 121.37, \mathrm{CH}, \mathrm{C} 3$; 128.14, $\mathrm{ArCH}, 2\left(\mathrm{C}^{\prime}\right.$ ', C6') -BPS; 128.17, $\mathrm{ArCH},\left(\mathrm{C}^{\prime}, \mathrm{C}^{\prime}\right)$-Bn; 128.54, $\mathrm{ArCH},(\mathrm{C} 4 ')$-Bn; 128.94, ArCH, (C4') -BPS; 130.21, 130.23, ArCH, (C3', C5') -Bn; 132.74, 133.23, ArC, 2(C1') BPS; 135.84, 136.01, ArCH, 2(C3', C5') -BPS; 137.33, ArC, (C1') -Bn; 145.58, CH, C2; 162.83, CO, C1. MS: $473.1\left([\mathrm{M}+\mathrm{H}]^{+}, 10 \%\right), 495.1\left([\mathrm{M}+\mathrm{Na}]^{+}, 100\right), 967.2\left([2 \mathrm{M}+\mathrm{Na}]^{+}, 15\right)$. HRMS calc'd for $\mathrm{C}_{29} \mathrm{H}_{32} \mathrm{O}_{4} \mathrm{Si}\left(\mathrm{Na}^{+}\right)$requires 495.197, found 495.196. Anal. Calc'd for $\mathrm{C}_{29} \mathrm{H}_{32} \mathrm{O}_{4} \mathrm{Si}$ (472.2): C, 73.69; H, 6.92. Found C, 73.33; H, 6.29. $[\alpha]_{\mathrm{D}}^{22}+15.47^{\circ}\left(\mathrm{c}=2.7, \mathrm{CHCl}_{3}\right)$.

\section{1,5-Anhydro-4-O-benzoyl-6-O-t-butyldiphenylsilyl-2,3-deoxy-1-oxo-D-arabino-hex-2-enitol}

(7). A solution of MCPBA ( $85 \%$ anhydrous, $0.04 \mathrm{~g}, 0.21 \mathrm{mmol}$ ) in $\mathrm{CH}_{2} \mathrm{Cl}_{2}(3 \mathrm{ml})$ was cooled to $-20^{\circ}$ and added to an equally cooled solution of $5(0.10 \mathrm{~g}, 0.22 \mathrm{mmol})$ in $\mathrm{CH}_{2} \mathrm{Cl}_{2}(3 \mathrm{ml})$. $\mathrm{BF}_{3} \cdot \mathrm{Et}_{2} \mathrm{O}(0.012 \mathrm{ml}, 0.013 \mathrm{~g}, 0.092 \mathrm{mmol})$ was added. The reaction was stirred at $-20^{\circ}$ for $3.5 \mathrm{hr}$ and quenched with saturated $\mathrm{NaHCO}_{3}$ solution $(10 \mathrm{ml})$. The aqueous phase was reextracted with $\mathrm{CH}_{2} \mathrm{Cl}_{2}(3 \mathrm{X} 10 \mathrm{ml})$, the organic phases were combined, dried $\left(\mathrm{MgSO}_{4}\right)$ and evaporated under reduced pressure to afford an orange solid (96 mg crude). Column chromatography $\left(\mathrm{SiO}_{2}, 3: 2\right.$ light petroleum/ $\left.\mathrm{CH}_{2} \mathrm{Cl}_{2}\right)$ gave the desired product as a brown oil $7\left(\mathrm{R}_{\mathrm{f}} 0.84\right.$ in 1:1, light petroleum/ ethyl acetate, $45.3 \mathrm{mg}, 57 \%)$. IR $\left(\mathrm{CH}_{2} \mathrm{Cl}_{2}\right): 3053 \mathrm{~s}, 2931 \mathrm{~s}, 2858 \mathrm{~s}, 1965 \mathrm{w}, 1905 \mathrm{w}$, $1820 \mathrm{w}, 1736 \mathrm{~s}, 1649 \mathrm{w}, 1602 \mathrm{~m}, 1588 \mathrm{~m}, 1472 \mathrm{~m}, 1452 \mathrm{~m}, 1428 \mathrm{~s}, 1390 \mathrm{~m}, 1316 \mathrm{~m}, 1266 \mathrm{~s}, 1112 \mathrm{~s}$, $1070 \mathrm{~m}, 1026 \mathrm{~m}, 977 \mathrm{~m}, 858 \mathrm{w}, 823 \mathrm{~m}, 738 \mathrm{~s}, 708 \mathrm{~s} \mathrm{~cm}^{-1} .{ }^{1} \mathrm{H}$ n.m.r. $\delta 1.02$, s, $9 \mathrm{H}, 3\left(\mathrm{CH}_{3}\right)$-BPS; 3.90 , 
d, J 4.0 Hz, 2H, $\left(\mathrm{CH}_{2}\right), \mathrm{H} 6 ; 4.7, \mathrm{q}, J 4.0 \mathrm{~Hz}, 1 \mathrm{H}, \mathrm{H} 5 ; 5.93, \mathrm{t}, J$ 4.8, 1H, H4; 6.14, d, J $10.9 \mathrm{~Hz}$, 1H, H2; 6.89, dd, J 10.1, 3.7, 1H, H3; 7.17-7.66, m, 13 ArH; 8.01, d, J 6.7, 2ArH, (H2', H4') -Bz. ${ }^{13} \mathrm{C}$ n.m.r. $\delta 19.22, \mathrm{CH}_{2}, \mathrm{C} 6 ; 26.75,3\left(\mathrm{CH}_{3}\right)$-BPS; 62.72, $\underline{\mathrm{C}}\left(\mathrm{CH}_{3}\right)_{3}$-BPS; 63.92, $\mathrm{CH}, \mathrm{C} 5 ; 80.22$, $\mathrm{CH}, \mathrm{C} 4 ; 123.02, \mathrm{CH}, \mathrm{C} 3 ; 127.68,127.75, \mathrm{ArCH}, 2\left(\mathrm{C}^{\prime}, \mathrm{C}^{\prime}\right)$ - $\mathrm{BPS} ; 128.47$, ArCH, (C2', C6') $\mathrm{Bz} ; 128.77, \mathrm{ArC},\left(\mathrm{C} 1^{\prime}\right)$-Bz; 129.74, $\mathrm{ArCH},(\mathrm{C} 4 ')$-Bz; 129.77, 129.83, ArCH, 2(C4') -BPS; 132.31, ArC, (C1') -BPS; 132.26, ArC, (C1') -BPS; 133.61, $\mathrm{ArCH},(\mathrm{C} 3 ', \mathrm{C} 5 ')$-Bz; 135.33, 135.47, ArCH, 2(C3', C5') -BPS; 141.93, CH, C2; 165.07, CO, C1. MS: $487.0\left([\mathrm{M}+\mathrm{H}]^{+}, 5 \%\right)$, $509.2\left([\mathrm{M}+\mathrm{Na}]^{+}, 100\right), 525.1\left([\mathrm{M}+\mathrm{K}]^{+}, 23\right), 995.2\left([2 \mathrm{M}+\mathrm{Na}]^{+}, 23\right)$. HRMS calc'd for $\mathrm{C}_{29} \mathrm{H}_{30} \mathrm{O}_{5} \mathrm{Si}\left(\mathrm{Na}^{+}\right)$requires 509.176 found 509.175. Anal. Calc'd for $\mathrm{C}_{29} \mathrm{H}_{30} \mathrm{O}_{5} \mathrm{Si}$ (486.6): C, 71.58; H 6.21. Found C, 71.54; H, 6.28. $[\alpha]_{\mathrm{D}}^{26}+106.9^{\circ}\left(\mathrm{c}=3, \mathrm{CHCl}_{3}\right)$.

\section{2,3-Bis-deoxy-2H-3-nitromethyl-4-O-benzyl-6-O-t-butyldiphenylsilyl-D-allonolactone (9a)} and 2,3-bis-deoxy-2H-3-nitromethyl-4-O-benzyl-6-O-t-butyldiphenylsilyl-D-gluconolactone (9b)

\section{Method 1}

A solution of the benzyl lactone $8(50.0 \mathrm{mg}, 0.11 \mathrm{mmol})$ in dry acetonitrile $(2 \mathrm{ml})$ was added to a solution of 18-crown-6 (0.0014 g, $0.005 \mathrm{mmol})$ and KF (12 mg, $0.002 \mathrm{mmol})$ in dry acetonitrile $(1 \mathrm{ml})$, followed by nitromethane $(114 \mu \mathrm{L}, 128.9 \mathrm{mg}, 2.11 \mathrm{mmol})$. It was refluxed, and once the reaction was complete $(1 \mathrm{hr})$ the solvent removed under vacuum, the residue dissolved in $\mathrm{CH}_{2} \mathrm{Cl}_{2}$ $(3 \mathrm{ml})$ and washed with dil. $\mathrm{HCl}(1 \mathrm{M}, 2 \times 3 \mathrm{ml})$. The organic extract was dried $\left(\mathrm{MgSO}_{4}\right)$ and reduced under pressure to afford an orange oil $(56 \mathrm{mg})$. The crude product was purified using flash column chromatography $\left(\mathrm{SiO}_{2}, 3: 1\right.$, light petroleum/ethyl acetate). The desired product in its isomer ratios of 9:2, 9a $\left(28 \mathrm{mg}, 51 \%, \mathrm{R}_{\mathrm{f}} 0.59\right)$ to $\mathbf{9 b}\left(6 \mathrm{mg}, 11 \%, \mathrm{R}_{\mathrm{f}} 0.51\right)$ respectively were isolated, in addition to $9 \mathrm{c}\left(6 \mathrm{mg}, 11 \%, \mathrm{R}_{\mathrm{f}} 0.05\right)$.

\section{Method 2}

A solution of the benzyl lactone $8(50.0 \mathrm{mg}, 0.11 \mathrm{mmol})$ in dry THF $(2 \mathrm{ml})$ was added to a solution of TBAF $(62 \mu \mathrm{L}, 0.002 \mathrm{mmol})$ in dry THF $(1 \mathrm{ml})$, followed by nitromethane $(114 \mu \mathrm{L}$, $128.9 \mathrm{mg}, 2.11 \mathrm{mmol})$. It was refluxed, and once the reaction was complete $(20 \mathrm{~min})$ the solvent removed under vacuum, the residue dissolved in $\mathrm{CH}_{2} \mathrm{Cl}_{2}(3 \mathrm{ml})$ and washed with dil. $\mathrm{HCl}(1 \mathrm{M}, 2$ $\mathrm{X} 3 \mathrm{ml})$. The organic extract was dried $\left(\mathrm{MgSO}_{4}\right)$ and reduced under pressure to afford an orange oil $(40 \mathrm{mg})$. The crude product was purified using flash column chromatography $\left(\mathrm{SiO}_{2}, 10: 1\right.$, light petroleum/ethyl acetate) to yield 9c $(20 \mathrm{mg}, 66 \%)$. 9a IR $\left(\mathrm{CH}_{2} \mathrm{Cl}_{2}\right): 3055 \mathrm{~m}, 2976 \mathrm{w}, 2956 \mathrm{~m}$, $2933 \mathrm{~m}, 2860 \mathrm{~m}, 1744 \mathrm{~s}, 1557 \mathrm{~s}, 1472 \mathrm{w}, 1428 \mathrm{~m}, 1371 \mathrm{w}, 1286 \mathrm{~s}, 1211 \mathrm{w}, 1182 \mathrm{w}, 1114 \mathrm{~m}, 1088 \mathrm{w}$, $1028 \mathrm{~m}, 940 \mathrm{w}, 896 \mathrm{w}, 823 \mathrm{w}, 738 \mathrm{~s}, 704 \mathrm{~s} \mathrm{~cm}^{-1} .{ }^{1} \mathrm{H}$ n.m.r. $\delta 1.06, \mathrm{~s}, 9 \mathrm{H}, 3\left(\mathrm{CH}_{3}\right)-\mathrm{BPS} ; 2.55,2.62$, ABX, J 17.64, 10.05, 7.74 Hz, 2H, $\mathrm{CH}_{2}, \mathrm{H} 2 ; 3.16-3.25, \mathrm{~m}, 1 \mathrm{H}, \mathrm{CH}, \mathrm{H} 3 ; 3.77,3.81, \mathrm{ABX}, J$ $11.19,5.89,4.41 \mathrm{~Hz}, 2 \mathrm{H}, \mathrm{CH}_{2}, \mathrm{H6} ; 3.92, \mathrm{t}, J 2.46 \mathrm{~Hz}, 1 \mathrm{H}, \mathrm{CH}, \mathrm{H} 4 ; 4.27,4,54, \mathrm{ABX}, J_{\text {a-e }} 13.20$ $\mathrm{Hz}, J_{\mathrm{a}-3} 7.65 \mathrm{~Hz}, J_{\mathrm{e}-3} 6.41 \mathrm{~Hz}, 2 \mathrm{H}, \mathrm{CH}_{2}, \mathrm{CH}_{2} \mathrm{NO}_{2} ; 4.54,4.56, \mathrm{ABq}, J 11.20 \mathrm{~Hz}, 2 \mathrm{H}, \mathrm{OCH}_{2} \mathrm{Ph}$; 4.64-4.66, m, 1H, CH, H5; 7.28-7.99, m, 15ArH. Irradiations: H4, d, J4,5 1.90 Hz. H4, d, J4,3 2.90 Hz. H5, d, $J_{5,4} 1.90$ Hz. ${ }^{13} \mathrm{C}$ n.m.r. $\delta 19.17, \mathrm{CH}_{2}, \mathrm{C} 6 ; 26.82,3\left(\mathrm{CH}_{3}\right)$-BPS; 29.84, $\mathrm{CH}_{2}, \mathrm{CH}_{2} \mathrm{NO}_{2}$; 32.84, $\mathrm{CH}, \mathrm{C} 3 ; 63.36, \underline{\mathrm{C}}\left(\mathrm{CH}_{3}\right)_{3}$-BPS; 70.86, $\mathrm{CH}, \mathrm{C} 5 ; 71.72, \mathrm{CH}_{2}, \mathrm{OC}_{2}{ }_{2} \mathrm{Ph} ; 75.30, \mathrm{CH}_{2}, \mathrm{C} 2$; 79.06, CH, C4; 127.90, 128.02, ArCH, 3(C2', C4') -Bn, BPS; 128.35, 128.63, ArCH, 2(C4') - 
BPS; 130.05, ArCH, (C3', C5') -Bn; 131.73, ArC, (C1') -BPS; 132.14, ArC, (C1') -BPS; 135.38, 135.51, ArCH, 2(C3', C5') -BPS; 136.40, ArC, (C1') -Bn; 167.61, CO, C1. MS: 532.3 $\left([\mathrm{M}]^{+}, 30 \%\right), 556.2\left([\mathrm{M}+\mathrm{Na}]^{+}, 60\right),\left(\left[\mathrm{M}+\mathrm{CH}_{3} \mathrm{NO}_{2}(\mathrm{H})\right]^{+}, 100\right), 1089.3,\left([2 \mathrm{M}+\mathrm{Na}]^{+}, 20\right)$. HRMS calc'd for $\mathrm{C}_{30} \mathrm{H}_{35} \mathrm{NO}_{6} \mathrm{Si}\left(\mathrm{Na}^{+}\right)$requires 556.213, found 556.213. $\mathrm{C}_{30} \mathrm{H}_{35} \mathrm{NO}_{6} \mathrm{Si}\left(\mathrm{K}^{+}\right)$requires 572.187, found 572.187. Anal. Calc'd for $\mathrm{C}_{30} \mathrm{H}_{35} \mathrm{NO}_{6} \mathrm{Si}$ (533.7): C 67.52; H6.61. Found C 67.01,

H 6.29. $[\alpha]_{\mathrm{D}}^{23}+7.66^{\circ}\left(\mathrm{c}=0.3, \mathrm{CHCl}_{3}\right)$. 9b This compound decomposed rapidly. IR $\left(\mathrm{CH}_{2} \mathrm{Cl}_{2}\right)$ : $3054 \mathrm{~m}, 2986 \mathrm{~m}, 2932 \mathrm{~m}, 2860 \mathrm{~m}, 1736 \mathrm{~s}, 1557 \mathrm{~s}, 1428 \mathrm{~s}, 1379 \mathrm{w}, 1266 \mathrm{~s}, 113 \mathrm{~m}, 1028 \mathrm{w}, 896 \mathrm{w}$, $823 \mathrm{w}, 739 \mathrm{~s}, 704 \mathrm{~s} \mathrm{~cm}^{-1} .{ }^{1} \mathrm{H}$ n.m.r. $\delta 1.08, \mathrm{~s}, 9 \mathrm{H}, 3\left(\mathrm{CH}_{3}\right)-\mathrm{BPS} ; 2.42-2.60, \mathrm{~m}, 2 \mathrm{H}, \mathrm{CH}_{2}, \mathrm{H} 2 ; 2.89$, td, $J_{3,4} 12.75 \mathrm{~Hz}, J_{3-2 \mathrm{a}} 6.02 \mathrm{~Hz}, J_{3-2 \mathrm{e}} 4.50 \mathrm{~Hz}, 1 \mathrm{H}, \mathrm{CH}, \mathrm{H} 3 ; 3.84$, dd (ABX), $J_{6 a-e} 12.0 \mathrm{~Hz}, J_{6 \mathrm{a}-5}$ $2.40 \mathrm{~Hz}, 1 \mathrm{H}, \mathrm{CH}$ of $\mathrm{CH}_{2}, \mathrm{H6} ; 3.97$, dd (ABX), $J_{6 \mathrm{e}-5} 1.80 \mathrm{~Hz}, 1 \mathrm{H}, \mathrm{CH}$ of $\mathrm{CH}_{2}, \mathrm{H} 6 ; 3.92, \mathrm{t}, \mathrm{J} 7.80$ $\mathrm{Hz}, 1 \mathrm{H}, \mathrm{CH}, \mathrm{H} 4 ; 4.24, \mathrm{td}, J_{5-4} 7.80 \mathrm{~Hz}, J_{5-6 \mathrm{a}} 2.10 \mathrm{~Hz}, J_{5-6 \mathrm{e}} 1.80 \mathrm{~Hz}, 1 \mathrm{H}, \mathrm{CH}, \mathrm{H} 5 ; 4.35,4,44, \mathrm{ABX}$, $J_{\text {a-e }} 12.60 \mathrm{~Hz}, J_{\mathrm{a}-3} 6.60 \mathrm{~Hz}, J_{\mathrm{e}-3} 4.80 \mathrm{~Hz}, 2 \mathrm{H}, \mathrm{CH}_{2}, \mathrm{CH}_{2} \mathrm{NO}_{2} ; 4.55, \mathrm{~s}, 2 \mathrm{H}, 0 \mathrm{C}_{2} \mathrm{Ph} ; 4.31-4.46, \mathrm{~m}$, 9ArH; 7.64-7.85, m, 15ArH. ${ }^{13} \mathrm{C}$ n.m.r. $\delta$ 19.11, C6, $\left(\mathrm{CH}_{2}\right) ; 26.96,3\left(\mathrm{CH}_{3}\right)-\mathrm{BPS} ; 31.71, \mathrm{CH}_{2}$, $\left(\mathrm{CH}_{2} \mathrm{NO}_{2}\right) ; 36.48, \mathrm{C} 3,(\mathrm{CH}) ; 62.78, \underline{\mathrm{C}}\left(\mathrm{CH}_{3}\right)_{3}$-BPS; 72.42, C5, $(\mathrm{CH}) ; 73.57, \mathrm{C} 2,\left(\mathrm{CH}_{2}\right)$; 76.13, $\mathrm{CH}_{2},\left(\mathrm{OC}_{2} \mathrm{Ph}\right) ; 79.91, \mathrm{C} 4,(\mathrm{CH}) ; 127.79,127.84, \mathrm{ArCH}, 2\left(\mathrm{C} 2 ', \mathrm{C}^{\prime}\right)$-BPS; 127.95, ArCH (C2', C6') -Bn; 128.36, ArCH, (C4') -Bn; 128.63, ArCH, 2(C4') -BPS; 129.95, ArCH, (C3', C5') -Bn; 133.99, ArC, 2(C1') -BPS; 135.49, 135.67, ArCH, 2(C3', C5') -BPS; 136.55, ArC, (C1') Bn; 155.27, C1, (CO). MS: $532.3\left([\mathrm{M}]^{+}, 80 \%\right), 533.3\left([\mathrm{M}+\mathrm{H}]^{+}, 35\right), 556.2\left([\mathrm{M}+\mathrm{Na}]^{+}, 20\right) .9 \mathrm{c}$ IR $\left(\mathrm{CH}_{2} \mathrm{Cl}_{2}\right): 3678 \mathrm{w}, 3600 \mathrm{w}, 3448 \mathrm{w}, 3055 \mathrm{~m}, 2987 \mathrm{~m}, 2931 \mathrm{~m}, 2360 \mathrm{w}, 2306 \mathrm{w}, 1740 \mathrm{~m}, 1557 \mathrm{~s}$, $1498 \mathrm{w}, 1422 \mathrm{~m}, 1383 \mathrm{w}, 1285 \mathrm{~s}, 1071 \mathrm{w}, 1028 \mathrm{w}, 896 \mathrm{w}, 740 \mathrm{~s}, 795 \mathrm{~cm}^{-1} .{ }^{1} \mathrm{H}$ n.m.r. $\delta(400 \mathrm{MHz})$ 1.50-2.20, bs, OH; 2.57, 2.63, ABX, J 17.47, 9.44, $3.27 \mathrm{~Hz}, 2 \mathrm{H}, \mathrm{CH}_{2}, \mathrm{H} 2 ; 3.14-3.22, \mathrm{~m}, 1 \mathrm{H}, \mathrm{CH}$, H3; 3.76, 3.91, ABX, $J_{6 \mathrm{a}-\mathrm{e}} 12.10 \mathrm{~Hz}, J_{6 \mathrm{a}-5} 4.56 \mathrm{~Hz}, J_{6 \mathrm{e}-5} 3.72 \mathrm{~Hz}, 2 \mathrm{H}, \mathrm{CH}_{2}, \mathrm{H6}$; 3.90, t, J 3.34, $3.27 \mathrm{~Hz}, 1 \mathrm{H}, \mathrm{CH}, \mathrm{H} 4 ; 4.58,4.62, \mathrm{ABX}, J_{\mathrm{e}-\mathrm{a}} 13.24 \mathrm{~Hz}, J_{\mathrm{e}-3} 6.61 \mathrm{~Hz}, J_{\mathrm{a}-3} 7.43 \mathrm{~Hz}, 2 \mathrm{H}, \mathrm{CH}_{2}$, $\mathrm{CH}_{2} \mathrm{NO}_{2} ; 4.58,4.62, \mathrm{ABq}, J 11.41 \mathrm{~Hz}, 2 \mathrm{H}, \mathrm{CH}_{2}, \mathrm{OCH}_{2} \mathrm{Ph} ; 4.58-4.60, \mathrm{~m}, 1 \mathrm{H}, \mathrm{CH}, \mathrm{H} 5 ;$ 7.27-7.65, m, 5ArH. ${ }^{13} \mathrm{C}$ n.m.r. $\delta$ 29.61, $\mathrm{CH}_{2}, \mathrm{C} 6 ; 33.17, \mathrm{CH}, \mathrm{C} 3 ; 62.29, \mathrm{CH}_{2}, \mathrm{CH}_{2} \mathrm{NO}_{2} ; 70.95, \mathrm{CH}, \mathrm{C} 5$; 72.04, $\mathrm{CH}_{2}, \mathrm{OCH}_{2} \mathrm{Ph} ; 75.11, \mathrm{CH}_{2}, \mathrm{C} 2 ; 79.79, \mathrm{CH}, \mathrm{C} 4 ; 128.06, \mathrm{ArCH}, 2\left(\mathrm{C} 2 ', \mathrm{C}^{\prime}\right), 128.40, \mathrm{ArCH}$, (C4'); 128.64, $\mathrm{ArCH},\left(\mathrm{C}^{\prime}\right.$ ', C5'); 136.35, ArC, (C1'); 163.66, CO, C1. MS: $318.1\left([\mathrm{M}+\mathrm{Na}]^{+}\right.$, $100 \%), 334.2\left([\mathrm{M}+\mathrm{K}]^{+}, 25\right), 629.3\left([2 \mathrm{M}+\mathrm{K}]^{+}, 25\right)$.

\section{2,3-Bis-deoxy-2H-3-phenylthio-4-O-benzyl-6-O-t-butyldiphenylsilyl-D-allonolactone (10a) and 2,3-bis-deoxy-2H-3-phenylthio-4-O-benzyl-6-O-t-butyldiphenylsilyl-D-gluconolactone (10b) \\ Method 1}

To a solution of the benzyl lactone $8(50.0 \mathrm{mg}, 0.11 \mathrm{mmol})$ in anhydrous THF $(2 \mathrm{ml})$, under a stream of nitrogen, TBAF $(0.56 \mu \mathrm{L} 0.002 \mathrm{mmol})$ was added whilst stirring at room temperature $\left(18^{\circ}\right)$, followed by thiophenol $(9.85 \mu \mathrm{L}, 0.10 \mathrm{mmol})$. After $1 \mathrm{hr}$ the solvent was evaporated in vacuo to give a colourless oil. The crude product was purified via flash column chromatography $\left(\mathrm{SiO}_{2}, 5: 1\right.$, light petroleum/ethyl acetate) to afford the desired product in its isomer ratios of 3:1, derivatives 10a $\left(25.2 \mathrm{mg}, 45 \%, \mathrm{R}_{\mathrm{f}} 0.76\right)$ to $10 \mathrm{~b}\left(8.40 \mathrm{mg}, 15 \%, \mathrm{R}_{\mathrm{f}} 0.65\left(2: 1, \mathrm{CH}_{2} \mathrm{Cl}_{2} /\right.\right.$ light petroleum), respectively. The lactone $8\left(7.5 \mathrm{mg}, 15 \%, \mathrm{R}_{\mathrm{f}} 0.54\right.$ in $2: 1, \mathrm{CH}_{2} \mathrm{Cl}_{2} /$ light petroleum) was also isolated. 10a IR $\left(\mathrm{CH}_{2} \mathrm{Cl}_{2}\right)$ : 3054m, 2955m, 2932m, 2860m, 1740s, 1589w, 1560w, 
$1543 \mathrm{~m}, 1473 \mathrm{~m}, 1439 \mathrm{~m}, 1428 \mathrm{~m}, 1379 \mathrm{w}, 1286 \mathrm{~s}, 1215 \mathrm{~m}, 1114 \mathrm{~s}, 1026 \mathrm{~m}, 942 \mathrm{w}, 896 \mathrm{w}, 823 \mathrm{~m}, 739 \mathrm{~s}$, $704 \mathrm{~s} \mathrm{~cm}^{-1} .{ }^{1} \mathrm{H}$ n.m.r $\delta$ 0.98, s, 9H, 3( $\left.\mathrm{CH}_{3}\right)$-BPS; 2.75, (dd) ABX, $J_{2 \mathrm{e}-3 \mathrm{e}} 5.49 \mathrm{~Hz}, J_{2 \mathrm{a}-3} 10.02 \mathrm{~Hz}$, $J_{2 \mathrm{e}-\mathrm{a}} 17.58 \mathrm{~Hz}, 1 \mathrm{H}, \mathrm{CH}$ of $\mathrm{CH}_{2}, \mathrm{H} 2 ; 2.84$, (dd), ABX, $J_{2 \mathrm{a}-3 \mathrm{e}} 10.02 \mathrm{~Hz}, J_{2 \mathrm{a} \text {-e }} 17.58 \mathrm{~Hz}, 1 \mathrm{H}, \mathrm{CH}$ of $\mathrm{CH}_{2}, \mathrm{H} 2 ; 3.76,3.80, \mathrm{ABX}, J_{6 \mathrm{e}-5 \mathrm{a}} 11.33 \mathrm{~Hz}, J_{6 \mathrm{a}-5} 17.24 \mathrm{~Hz}, J_{6 \mathrm{a}-\mathrm{e}} 20.46 \mathrm{~Hz}, 2 \mathrm{H}, \mathrm{CH}_{2}, \mathrm{H6}$; 3.863.96, m, 1H, CH, H3; 4.14, t, J4e-3e $3.16 \mathrm{~Hz}, J_{4 \mathrm{e}-5 \mathrm{e}} 3.57 \mathrm{~Hz}, 1 \mathrm{H}, \mathrm{H} 4 ; 4.58-4.62, \mathrm{~m}, 1 \mathrm{H}, \mathrm{CH}, \mathrm{H} 5$; 4.62, 4.71, ABq, J $11.53 \mathrm{~Hz}, 2 \mathrm{H}, \mathrm{OC}_{2} \underline{\mathrm{Ph}} ; 7.25-7.49, \mathrm{~m}, 12 \mathrm{ArH} ; 7.56-7.59, \mathrm{~m}, 8 \mathrm{ArH}$. Irradiations: $\mathrm{H} 3$, dd $J_{3-2} 5.49,10.02 \mathrm{~Hz}$. H5, q, $J_{5-6} 11.54,8.93 \mathrm{~Hz} . \mathrm{H} 5 \mathrm{~d}, J_{5-4} 3.6 \mathrm{~Hz} .{ }^{13} \mathrm{C}$ n.m.r. $\delta$ 19.22, $\mathrm{CH}_{2}, \mathrm{C} 6 ; 26.87,3\left(\underline{\mathrm{CH}}_{3}\right)$-BPS; 33.96, $\mathrm{CH}_{2}, \mathrm{C} 2 ; 44.13, \mathrm{CH}, \mathrm{C} 3 ; 65.23 \underline{\mathrm{C}}\left(\mathrm{CH}_{3}\right)_{3}-\mathrm{BPS}$; 72.41, $\mathrm{CH}_{2},\left(\mathrm{OCH}_{2} \mathrm{Ph}\right) ; 74.36, \mathrm{CH}, \mathrm{C} 5 ; 80.15 \mathrm{CH}, \mathrm{C} 4 ; 128.03,128.06, \mathrm{ArCH}, 2(\mathrm{C} 2 ', \mathrm{C} 6$ ') -BPS; 128.19, ArCH, (C2', C6') -Bn; 128.32, ArCH, (C2', C6') -SPh; 128.62, 129.40, 130.14, ArCH, 4(C4'); 132.03, ArC, (C1'); 132.69, ArC, (C1'); 133.05, ArCH, 2(C3', C5') -Bn, SPh; 133.37 , $\mathrm{ArC},\left(\mathrm{Cl}^{\prime}\right.$ '); 135.54, 135.88, $\mathrm{ArCH}, 2(\mathrm{C} 3$ ', C5') -BPS; 137.27, $\mathrm{ArC}, \mathrm{C} 1$ '; 166.50, C1, CO. MS: $605.3\left([\mathrm{M}+\mathrm{Na}]^{+} 100 \%\right), 621.2\left([\mathrm{M}+\mathrm{K}]^{+} 28\right)$. HRMS calc'd for $\mathrm{C}_{35} \mathrm{H}_{38} \mathrm{O}_{4} \mathrm{SSi}\left(\mathrm{Na}^{+}\right)$requires 605.216, found 605.217. $\mathrm{C}_{35} \mathrm{H}_{38} \mathrm{O}_{4} \mathrm{SSi}\left(\mathrm{K}^{+}\right)$requires 621.190, found 621.189. $[\alpha]_{\mathrm{D}}^{23}+29.6^{\circ}(\mathrm{c}=$ 0.25, $\left.\mathrm{CHCl}_{3}\right)$. 10b IR $\left(\mathrm{CH}_{2} \mathrm{Cl}_{2}\right): 3645 \mathrm{w}, 3466 \mathrm{w}, 2985 \mathrm{~s}, 2942 \mathrm{~s}, 2909 \mathrm{~s}, 2086 \mathrm{w}, 1890 \mathrm{w}, 1750 \mathrm{~s}$, $1448 \mathrm{~s}, 1373 \mathrm{~s}, 1301 \mathrm{~s}, 1232 \mathrm{~s}, 1160 \mathrm{w}, 1098 \mathrm{~s}, 1047 \mathrm{~s}, 938 \mathrm{~m}, 918 \mathrm{~m}, 847 \mathrm{~m}, 787 \mathrm{w}, 743 \mathrm{w}, 705 \mathrm{~m} \mathrm{~cm}^{-1}$. ${ }^{1} \mathrm{H}$ n.m.r. $\delta$ 1.07, s, 9H, 3( $\left.\mathrm{CH}_{3}\right)$-BPS; 2.63, (dd)- ABX, $J_{2 \mathrm{a}-2 \mathrm{e}} 16.62 \mathrm{~Hz}, J_{2 \mathrm{e}-3} 5.37 \mathrm{~Hz}, 1 \mathrm{H}, \mathrm{CH}$ of $\mathrm{CH}_{2}, \mathrm{H} 2 ; 3.02$ (dd)- $\mathrm{ABX}, J_{2 \mathrm{a}-3} 6.73 \mathrm{~Hz}, 1 \mathrm{H}, \mathrm{CH}$ of $\mathrm{CH}_{2}, \mathrm{H} 2 ; 3.66, \mathrm{bq}, J_{3-4} 5.63 \mathrm{~Hz}, J_{3-5} 12.09$ $\mathrm{Hz}, 1 \mathrm{H}, \mathrm{CH}, \mathrm{H} 3$; 3.91, qd, J6a-e 21.79, $J_{6 \mathrm{e}-5} 11.49 \mathrm{~Hz}, \mathrm{~Hz}, J_{6 \mathrm{a}-5} 2.70 \mathrm{~Hz}, 2 \mathrm{H}, \mathrm{CH}_{2}, \mathrm{H6}$; 4.04, dd, $J_{4 \mathrm{e}-5 \mathrm{e}} 7.97 \mathrm{~Hz}, J_{4 \mathrm{e}-3 \mathrm{a}} 5.63 \mathrm{~Hz}, 1 \mathrm{H}, \mathrm{CH}, \mathrm{H} 4 ; 4.21 \mathrm{td}, J_{5 \mathrm{e}-4 \mathrm{e}} 7.97 \mathrm{~Hz}, J_{5 \mathrm{e}-6 \mathrm{a}} 5.63 \mathrm{~Hz}, J_{5 \mathrm{e}-6 \mathrm{e}} 2.75 \mathrm{~Hz}$, 1H, CH, H5; 4.59, 4.82, ABq, J 10.99 Hz, 2H, OC$\underline{H}_{2} \mathrm{Ph} ; 7.28-7.69$, m, 20ArH. ${ }^{13} \mathrm{C}$ n.m.r. $\delta 19.37$, $\mathrm{CH}_{2}, \mathrm{C} 6 ; 26.93,3\left(\underline{\mathrm{CH}}_{3}\right)$-BPS; 34.55, $\mathrm{CH}_{2}, \mathrm{C} 2 ; 45.49, \mathrm{CH}, \mathrm{C} 3 ; 62.65 \underline{\mathrm{C}}\left(\mathrm{CH}_{3}\right)_{3}-\mathrm{BPS} ; 73.39, \mathrm{CH}_{2}$, $\mathrm{OCH}_{2} \mathrm{Ph} ; 75.06, \mathrm{CH}, \mathrm{C} 5 ; 81.11, \mathrm{CH}, \mathrm{C} 4 ; 127.70,127.73, \mathrm{ArCH}, 2\left(\mathrm{C} 2 ', \mathrm{C}^{\prime}\right)-\mathrm{BPS} ; 127.90$, 128.01, 128.13, 128.30, ArCH, 2(C2', C6') -Bn, SPh; 128.40, 129.26, $\mathrm{ArCH}, 2\left(\mathrm{C}^{\prime}\right)$ - Bn, SPh; 129.77, 129.78, ArCH, (C4') -BPS; 132.24, 132.34, 132.94, 3ArC, 3(C1'); 133.44, ArCH, 2(C3', C5') -Bn, SPh; 135.51, 135.61, 135.68, ArCH, 2(C3', C5'); 137.00, ArC, C1'; 169.24, C1, (CO). MS: $605.4\left([\mathrm{M}+\mathrm{Na}]^{+}, 50 \%\right), 621.2\left([\mathrm{M}+\mathrm{K}]^{+}, 100\right)$. HRMS calc'd for $\mathrm{C}_{35} \mathrm{H}_{38} \mathrm{O}_{4} \mathrm{SSi}\left(\mathrm{Na}^{+}\right)$ requires 605.216 , found $605.214 . \mathrm{C}_{35} \mathrm{H}_{38} \mathrm{O}_{4} \mathrm{SSi}\left(\mathrm{K}^{+}\right)$requires 621.190 , found 621.185 . $[\alpha]_{D}^{23}$ $+9.33^{\circ}\left(\mathrm{c}=0.45, \mathrm{CHCl}_{3}\right)$.

\section{2,3-Bis-deoxy-2H-3-benzylamino-4-O-benzyl-6-O-t-butyldiphenylsilyl-D-allonolactone (11a) and 2,3-bis-deoxy-2H-3-benzylamino-4-O-benzyl-6-O-t-butyldiphenylsilyl-D-gluconolactone (11b)}

\section{Method 1}

A solution of the benzyl lactone $8(100 \mathrm{mg}, 0.21 \mathrm{mmol})$ in dry methanol $(1 \mathrm{ml})$ was treated with freshly distilled benzylamine $(22.6 \mathrm{mg}, 23.0 \mu \mathrm{L}, 0.21 \mathrm{mmol})$ and left stirring at room temperature. The reaction was monitored by t.l.c and once complete $(24 \mathrm{hr})$, quenched with water $(5 \mathrm{ml})$ and extracted with $\mathrm{Et}_{2} \mathrm{O}(2 \mathrm{X} 10 \mathrm{ml})$. The organic extracts were combined, dried $\left(\mathrm{MgSO}_{4}\right)$ and concentrated to give a colourless oil $(112 \mathrm{mg})$. The crude product was purified by 
flash column chromatography $\left(\mathrm{SiO}_{2}, 5: 1\right.$, light petroleum/ethyl acetate) to yield the desired product in its isomer ratios of 2:3, compounds $11 b\left(49 \mathrm{mg}, 40 \%, \mathrm{R}_{\mathrm{f}} 0.33\right)$ and $11 \mathrm{a}(73 \mathrm{mg}, 60 \%$, $\mathrm{R}_{\mathrm{f}} 0.28$ ) respectively.

\section{Method 2}

A solution of the benzyl lactone $8(52.0 \mathrm{mg}, 0.11 \mathrm{mmol})$ in dry methanol $(2 \mathrm{ml})$ was treated with freshly distilled benzylamine $(33.0 \mathrm{mg}, 36.0 \mu \mathrm{L}, 0.33 \mathrm{mmol})$ and subjected to reflux. The reaction was monitored via t.l.c and once complete $(8 \mathrm{hr})$, quenched with water $(5 \mathrm{ml})$ and extracted with $\mathrm{Et}_{2} \mathrm{O}(2 \mathrm{X} 10 \mathrm{ml})$. The organic extracts were combined, dried $\left(\mathrm{MgSO}_{4}\right)$ and concentrated to give a colourless oil $(78 \mathrm{mg})$. The crude product was purified by flash column chromatography $\left(\mathrm{SiO}_{2}, 3: 1\right.$, light petroleum/ethyl acetate) to yield the desired product in one of its isomer forms 11a (57 mg, 92\%).

\section{Method 3}

A solution of the benzyl lactone $8(73.0 \mathrm{mg}, 0.15 \mathrm{mmol})$ in dry methanol $(2 \mathrm{ml})$ was treated with freshly distilled benzylamine $(16.5 \mathrm{mg}, 16.8 \mu \mathrm{L}, 0.15 \mathrm{mmol})$ and left stirring for $4 \mathrm{hr}$ at $0^{\circ}$ and then placed in the fridge for $18 \mathrm{hr}$. The reaction was quenched with water $(5 \mathrm{ml})$ and extracted with $\mathrm{Et}_{2} \mathrm{O}(2 \mathrm{X} 5 \mathrm{ml})$. The organic extracts were combined, dried $\left(\mathrm{MgSO}_{4}\right)$ and concentrated to give a colourless oil $(120 \mathrm{mg})$. The crude product was purified by flash column chromatography $\left(\mathrm{SiO}_{2}, 5: 1\right.$, light petroleum/ethyl acetate) to yield the desired product in its isomer ratios of 2:1 11b (54.0 mg, 60\%) and 11a (27 mg, 30\%).

\section{Method 4}

A solution of the benzyl lactone $8(73.0 \mathrm{mg}, 0.15 \mathrm{mmol})$ in dry methanol $(2 \mathrm{ml})$ was treated with freshly distilled benzylamine $(16.5 \mathrm{mg} 16.8 \mu \mathrm{L}, 0.15 \mathrm{mmol})$ and left stirring for $6 \mathrm{hr}$ at $-78^{\circ}$ and left in the freezer for 18 days. The reaction was quenched with water $(5 \mathrm{ml})$ and extracted with $\mathrm{Et}_{2} \mathrm{O}(2 \mathrm{X} 10 \mathrm{ml})$. The organic extracts were combined, dried $\left(\mathrm{MgSO}_{4}\right)$ and concentrated to give a colourless oil $(90 \mathrm{mg})$. The crude product was purified by flash column chromatography $\left(\mathrm{SiO}_{2}\right.$, $5: 1$, light petroleum/ethyl acetate) to yield the desired product in its isomer ratios of $5: 1, \mathbf{1 1 b}$ (30.0 $\mathrm{mg}, 34 \%)$ and 11a $(6.3 \mathrm{mg}, 7 \%)$, in addition to recovered $8(21.9 \mathrm{mg}, 30 \%)$. 11a IR $\left(\mathrm{CH}_{2} \mathrm{Cl}_{2}\right): 3306 \mathrm{~m}, 3052 \mathrm{~m}, 2931 \mathrm{~s}, 2858 \mathrm{~s}, 1652 \mathrm{~m}, 1588 \mathrm{w}, 1545 \mathrm{~m}, 1496 \mathrm{~m}, 1472 \mathrm{~m}, 1455 \mathrm{~s}, 1428 \mathrm{~s}$, $1391 \mathrm{w}, 1362 \mathrm{w}, 1266 \mathrm{~s}, 1189 \mathrm{w}, 1113 \mathrm{~s}, 1029 \mathrm{~m}, 1008 \mathrm{w}, 911 \mathrm{w}, 824 \mathrm{~m}, 738 \mathrm{~s}, 701 \mathrm{~s} \mathrm{~cm}^{-1} .{ }^{1} \mathrm{H}$ n.m.r. $\delta$ $1.09, \mathrm{~s}, 9 \mathrm{H}, 3\left(\mathrm{CH}_{3}\right)$-BPS; 2.47, 2.56, ABX, $J_{2 \mathrm{a}-\mathrm{e}} 15.52 \mathrm{~Hz} J_{2 \mathrm{a}-3} 7.84 \mathrm{~Hz}, J_{2 \mathrm{e}-3 \mathrm{e}} 4.16 \mathrm{~Hz}, 2 \mathrm{H}, \mathrm{CH}_{2}$, H2; 2.58-2.94, bs, NH; 3.34, bp, J 3.92, 7.79 Hz, 1H, CH, H3; 3.64-3.70, m, 1H, CH, H4; 3.73,

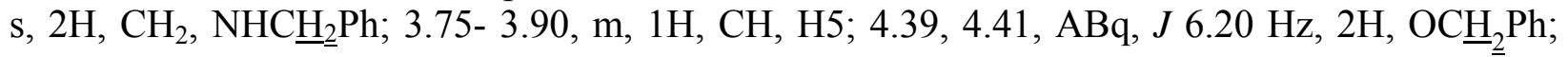
4.30-4.60, m, 2H, $\mathrm{CH}_{2}, \mathrm{H} 6 ; 7.05-7.70, \mathrm{~m}, 20 \mathrm{H}, 20 \mathrm{ArH}$. Irradiations: $\mathrm{H} 3, \mathrm{~d}, J_{3-4} 3.0 \mathrm{~Hz}$. H4, t, J4-5

6.2 Hz. ${ }^{13} \mathrm{C}$ n.m.r. $\delta$ 19.39, $\mathrm{CH}_{2}, \mathrm{C} 6 ; 27.03,3\left(\underline{\mathrm{CH}}_{3}\right)$-BPS; 36.30, $\mathrm{CH}_{2}, \mathrm{C} 2 ; 51.22, \mathrm{CH}_{2}$, $\left(\mathrm{NHCH}_{2} \mathrm{Ph}\right) ; 56.42, \mathrm{CH}, \mathrm{C} 3 ; 65.33, \underline{\mathrm{C}}\left(\mathrm{CH}_{3}\right)_{3}$-BPS; 72.81, CH, C5; 73.64, $\underline{\mathrm{C}}_{2} \mathrm{Ph} \mathrm{C6} ; 78.55$, CH, C4; 127.24, 127.55, 127.70, 127.81, 128.17, 128.33, 128.36, 128.51, ArCH, 4(C2', C4'); 129.74, ArCH, 2(C3', C5') -Bn, NHPh; 132.81, ArC, (C1') -BPS; 133.04, ArC, (C1') -BPS; 135.47, 135.54, ArCH, 2(C3', C5') -BPS; 137.82, ArC, (C1'); 138.3, ArC, C1'; 171.91, CO, C1. MS: $580.29\left([\mathrm{M}+\mathrm{H}]^{+}, 5 \%\right), 687.36\left(\left[\mathrm{M}+\mathrm{BnNH}_{3}\right]^{+}, 60\right)$. HRMS calc'd for $\mathrm{C}_{36} \mathrm{H}_{41} \mathrm{NO}_{4} \mathrm{Si}\left(\mathrm{H}^{+}\right)$ 
requires 580.288 found $580.289, \mathrm{C}_{36} \mathrm{H}_{41} \mathrm{NO}_{4} \mathrm{Si}\left(\mathrm{BnNH}_{3}{ }^{+}\right)$requires 687.362 found $687.361 .[\alpha]_{\mathrm{D}}^{22}{ }^{-}$ $7.83^{\circ}\left(\mathrm{c}=1, \mathrm{CHCl}_{3}\right)$ 11b IR $\left(\mathrm{CH}_{2} \mathrm{Cl}_{2}\right): 3056 \mathrm{~m}, 2933 \mathrm{~s}, 2932 \mathrm{~s}, 2845 \mathrm{~s}, 1748 \mathrm{~s}, 1472 \mathrm{~m}, 1428 \mathrm{~m}$, $1263 \mathrm{~m}, 1113 \mathrm{~s}, 1030 \mathrm{~m}, 823 \mathrm{~m}, 740 \mathrm{~s}, 782 \mathrm{~s} \mathrm{~cm}^{-1} .{ }^{1} \mathrm{H}$ n.m.r. $\delta 1.03, \mathrm{~s}, 9 \mathrm{H}, 3\left(\mathrm{CH}_{3}\right)$-BPS; $2.61,2.69$ ABX, $J_{2 \mathrm{a}-\mathrm{e}} 15.87 \mathrm{~Hz}, J_{2 \mathrm{a}-3} 6.04 \mathrm{~Hz}, J_{2 \mathrm{e}-3} 5.36 \mathrm{~Hz}, 2 \mathrm{H}, \mathrm{CH}_{2}, \mathrm{H} 2 ; 2.82-3.02, \mathrm{bs}, \mathrm{NH} ; 3.29, \mathrm{bp}, J$ $5.91 \mathrm{~Hz}, 11.40 \mathrm{~Hz}, 1 \mathrm{H}, \mathrm{CH}, \mathrm{H} 3: 3.51-3.83, \mathrm{~m}, 4 \mathrm{H}, 2(\mathrm{CH}) \mathrm{H} 4, \mathrm{H} 5$ and $1\left(\mathrm{CH}_{2}\right), \mathrm{H} 6 ; 3.56, \mathrm{~s}, 2 \mathrm{H}$, $\mathrm{CH}_{2}, \mathrm{NHC}_{2} \mathrm{Ph} ; 4.48,4.52, \mathrm{ABq}, J 11.06 \mathrm{~Hz}, 2 \mathrm{H}, \mathrm{OC}_{2}{ }_{2} \mathrm{Ph} ; 7.07-7.10, \mathrm{~m}, 2 \mathrm{ArH} ; 7.12-7.38, \mathrm{~m}$, 14ArH, 7.56-7.67, m, 4ArH. ${ }^{13} \mathrm{C}$ n.m.r. $\delta$ 19.45, $\mathrm{CH}_{2}, \mathrm{C} 6 ; 27.08,3\left(\mathrm{CH}_{3}\right)-\mathrm{BPS} ; 34.55, \mathrm{C} 2,\left(\mathrm{CH}_{2}\right)$; 51.38, $\mathrm{CH}_{2},\left(\mathrm{NH}_{\mathrm{CH}} \mathrm{Ph}\right) ; 57.11, \mathrm{CH}, \mathrm{C} 3 ; 65.19, \underline{\mathrm{C}}\left(\mathrm{CH}_{3}\right)_{3}-\mathrm{BPS} ; 73.86, \mathrm{CH}_{2}, \mathrm{OCH} 2 \mathrm{Ph} ; 74.27$, $\mathrm{CH}, \mathrm{C} 5 ; 78.72, \mathrm{CH}, \mathrm{C} 4 ; 127.31,127.83,127.87,127.91,127.98,128.41,128.46,128.59$, ArCH, 4(C2', C6'); 129.84, ArCH, 2(C3', C5') -Bn; 133.21, $\mathrm{ArC},\left(\mathrm{C} 1^{\prime}\right) ; 133.50, \mathrm{ArC},(\mathrm{C} 1$ '); 135.34, 135.77, 135.81, 135.89, ArCH, 2(C3', C5') -Bn; 138.21, ArC, (C1'); 139.67, ArC, (C1'); 173.09, $\mathrm{CO}, \mathrm{C} 1 . \mathrm{MS}: 578.3\left([\mathrm{M}]^{+}, 15 \%\right), 596.3\left(\left[\mathrm{M}+\mathrm{NH}_{3}\right]^{+}, 47\right), 610.4\left([\mathrm{M}+\mathrm{MeOH}]^{+}, 100\right)$.

\section{References}

1. $\quad$ Paquette, L.; Oplinger, J. J. Org. Chem. 1988, 53, 2953.

2. Ireland, R.; Wuts, P.; Ernst, B. J. Am. Chem. Soc. 1981, 103, 3205.

3. $\quad$ Bouzide, A.; Sauve, G. Tetrahedron Lett. 1997, 38, 5945.

4. Lichtenthaler, F.; Ronninger, S.; Jarglis, P. Liebigs Ann. Chem. 1989, 1153.

5. Perlmutter, P. Conjugate Addition Reactions in Organic Synthesis, Pergamon Press, 1992.

6. Kuwajima, I.; Murofushi, T.; Nakamura, E. Synthesis, 1976, 602.

7. Kambe, S.; Yasuda, H. Bull. Chem. Soc. Jpn. 1966, 39, 2549.

8. Belsky, I. J. Chem. Soc., Chem. Commun. 1977, 237.

9. Herradon, B.; Fenude, E.; Bao, R.; Valverde, S. J. Org. Chem. 1996, 61, 1143.

10. Furniss, B.; Hannaford, A.; Rogers, V.; Smith, P.; Tatchell, A. Vogel's Textbook of Practical Organic Chemistry, Longman (Fourth Edition), 1978, p 334. 